Abstract 59 Table 2 Performance of mTBI discharge decision rule and BIG criteria

\begin{tabular}{|c|c|c|c|}
\hline \multicolumn{4}{|c|}{ mTBI Risk Score } \\
\hline $\mathrm{N}=961$ & Deteriorated & No deterioration & \\
\hline Risk $=0$ (discharge & 0 & $34(3.5 \%)$ & Sensitivity $100 \%$ (95\% \\
\hline ED) & & & $\mathrm{Cl}: 97 \%$ to $100 \%)$ \\
\hline Risk >0 (Inpatient & 234 & 693 & Specificity $4.7 \%$ (95\% \\
\hline admission) & & & $\mathrm{Cl}: 3.3 \%$ to $6.5 \%)$ \\
\hline \multicolumn{4}{|c|}{ BIG Criteria } \\
\hline $\mathrm{N}=921$ & Deteriorated & No deterioration & \\
\hline \multirow[t]{2}{*}{ BIG 1 (discharge ED) } & 12 & 93 & Sensitivity $94 \%$ (95\% \\
\hline & & & Cl: $90.5 \%$ to $97 \%)$ \\
\hline BIG 2/3 (Inpatient & 210 & 606 & Specificity $13.3 \%$ (95\% \\
\hline admission) & & & Cl: $10.9 \%$ to $16.1 \%)$ \\
\hline
\end{tabular}

none of whom deteriorated. The decision rule outperformed the BIG criteria, which is used to triage hospital admissions in the USA.

External validation shows our decision rule may be safe for routine use in clinical practice. The inclusion of biomarkers or other novel factors may improve the calibration of the model and the specificity of the decision rule.

\section{Free papers}

\section{PREHOSPITAL DETERMINANTS OF SUCCESSFUL RESUSCITATION AFTER TRAUMATIC AND NON- TRAUMATIC OUT-OF-HOSPITAL CARDIAC ARREST}

${ }^{1}$ Ed Barnard, ${ }^{1}$ Daniel Sandbach, ${ }^{2}$ Tracy Nicholls, ${ }^{1}$ Alastair Wilson, ${ }^{1}$ Ari Ercole. ${ }^{1}$ East Anglian Air Ambulance; ${ }^{2}$ East of England Ambulance Service NHS Trust

\subsection{6/emj-2020-rcemabstracts.6}

Aims/Objectives/Background Out-of-hospital cardiac arrest (OHCA) is prevalent in the UK. Reported survival is lower than in countries with comparable healthcare systems; a better understanding of outcome determinants may identify areas for improvement. Aim: to compare differential determinants of survival to hospital admission and survival to hospital discharge for traumatic (TCA) and non-traumatic cardiac arrest (NCTA).

Methods/Design An analysis of 9109 OHCA in East of England between 1 January 2015 and 31 July 2017. Univariate descriptives and multivariable analysis were used to understand the determinants of survival for NTCA and TCA. Two Utstein outcome variables were used: survival to hospital admission and hospital discharge. Data reported as number (percentage), number (percentage $(95 \% \mathrm{CI})$ ) and median (IQR) as appropriate. Continuous data have been analysed with a Mann-Whitney $U$ test, and categorical data have been analysed with a $\chi 2$ test. Analyses were performed using the $\mathrm{R}$ statistical programming language.

Results/Conclusions The incidence of OHCA was 55.1 per 100000 population/year. The overall survival to hospital admission was $27.6 \%(95 \% \mathrm{CI} 26.7 \%$ to $28.6 \%)$ and the overall survival to discharge was $7.9 \%$ (95\%CI $7.3 \%$ to $8.5 \%)$. Survival to hospital admission and survival to hospital discharge were both greater in the NTCA group compared with the TCA group: $27.9 \%$ vs $19.3 \% \mathrm{p}=0.001$, and $8.0 \%$ vs $3.8 \% \mathrm{p}=0.012$ respectively.

Determinants of NTCA and TCA survival were different, and varied according to the outcome examined. In NTCA, bystander cardiopulmonary resuscitation (CPR) was associated with survival at discharge but not at admission, and the likelihood of bystander-CPR was dependent on geographical socioeconomic status.

NTCA and TCA are clinically distinct entities with different predictors for outcome and should be reported separately. Determinants of survival to hospital admission and discharge differ in a way that likely reflects the determinants of neurological injury. Bystander CPR public engagement may be best focused in more deprived areas.

\section{ESTABLISHING INJURY SURVEILLANCE IN EMERGENCY DEPARTMENTS IN NEPAL: EPIDEMIOLOGY AND BURDEN OF PAEDIATRIC INJURIES}

${ }^{1}$ Dan Magnus, ${ }^{2}$ Santosh Bhatta, ${ }^{2}$ Julie Mytton. ${ }^{1}$ Bristol Royal Hospital for Children; ${ }^{2}$ University of the West of England (UWE Bristol)

10.1136/emj-2020-rcemabstracts. 7

Aims/Objectives/Background Globally, injuries cause more than 5 million deaths annually. Children and young people are a particularly vulnerable group and injuries are the leading cause of death in people aged 5-24 years globally and a leading cause of disability.

In most low and middle-income countries where the majority of global child injury burden occurs, systems for routinely collecting injury data are limited. There is a continuing need for better data on childhood injuries and for injury surveillance.

The aim of our study was to introduce a hospital-based injury surveillance tool - the first of its kind in Nepal and explore its feasibility. We undertook prospective collection of data on all injuries/trauma presenting to 2 hospital emergency departments to describe the epidemiology of paediatric hospital injury presentations and associated risk factors.

Methods/Design A new injury surveillance system for use in emergency departments in Nepal was designed and used to collect data on patients presenting with injuries. Data were collected prospectively in two hospitals $24 \mathrm{~h}$ a day over 12 months (April 2019 - March 2020) by trained data collectors using tablet computers.

Results/Conclusions The total number of ED patients with injury in the study was 10,154 .

2,696 were patients aged $<18$ years. Most injuries in children were unintentional and over half of children presenting with injuries were $<10$ years of age. Falls, animal bites/stings and road traffic injuries accounted for nearly $75 \%$ of all injuries with some (drowning, poisonings and burns) under-represented. Over half of injuries were cuts, bites and open wounds. The next most common injury types were superficial injuries (14.2\%); fractures $(11.1 \%)$; sprains/dislocations (9.0\%). Child mortality was $1 \%$.

This is the biggest prospective injury surveillance study in a low or middle country in recent years and supports the use 